\title{
A graphical user interface for interactive searching strategies in discrete vector optimization
}

\author{
Walter Habenicht, Universität Hohenheim \\ Ralph Scheubrein, Universität Hohenheim
}

Interactive approaches are very powerfull tools in vector optimization problems. The system ENUQUAD is devoted to discrete vector optimization, namely to those problems with a large number of alternatives and a limited number of criteria. Problems of this type often occur in multicriteria combinatorial problems. ENUQUAD determines the set of efficient solutions of the problem and provides the user with a set of searching options like

- Reference point approach,

- Aspiration levels,

- Neighborhood scanning,

- Filtering approaches.

The aim of this paper is to describe the graphical user interface of the system, which is a user oriented one, too. It provides the user with a set of options concerning the presentation of the relevant information about the efficient set.

The whole system can be interpreted as a tool-box, which can be used by a decisionmaker to build up his individual searching strategy using graphical representations according to his choice. 